Review Article

\title{
Endoplasmic Reticulum Stress in Diabetic Nephrology: Regulation, Pathological Role, and Therapeutic Potential
}

\author{
Lihua Ni $\mathbb{D}^{1}$, Cheng Yuan $\mathbb{D}^{2}{ }^{2}$ and Xiaoyan $\mathrm{Wu} \mathbb{D}^{1}$ \\ ${ }^{1}$ Department of Nephrology, Zhongnan Hospital of Wuhan University, Wuhan 430071, China \\ ${ }^{2}$ Department of Gynecological Oncology, Zhongnan Hospital of Wuhan University, Wuhan 430071, China \\ Correspondence should be addressed to Xiaoyan Wu; wuxiaoyan_kid@163.com
}

Received 6 May 2021; Revised 29 June 2021; Accepted 17 July 2021; Published 3 August 2021

Academic Editor: Andrej Veljkovic

Copyright ( 92021 Lihua Ni et al. This is an open access article distributed under the Creative Commons Attribution License, which permits unrestricted use, distribution, and reproduction in any medium, provided the original work is properly cited.

\begin{abstract}
Recent progress has been made in understanding the roles and mechanisms of endoplasmic reticulum (ER) stress in the development and pathogenesis of diabetic nephropathy (DN). Hyperglycemia induces ER stress and apoptosis in renal cells. The induction of ER stress can be cytoprotective or cytotoxic. Experimental treatment of animals with ER stress inhibitors alleviated renal damage. Considering these findings, the normalization of ER stress by pharmacological agents is a promising approach to prevent or arrest DN progression. The current article reviews the mechanisms, roles, and therapeutic aspects of these findings.
\end{abstract}

\section{Introduction}

Diabetic nephropathy (DN) is one of the common microvascular complications of diabetes. The main clinical manifestations are proteinuria, hyperglycemia, and impaired renal function. Additionally, mesangial hyperplasia, glomerular sclerosis, extracellular matrix accumulation, and tubulointerstitial fibrosis can be observed pathologically. Numerous studies have demonstrated the role of endoplasmic reticulum (ER) stress in the pathogenesis of DN [1-3].

The ER is an important subcellular organ in eukaryotic cells. It facilitates the synthesis and export of proteins and lipids [4]. It can fold proteins in the cisternae and transport synthesized proteins to the Golgi apparatus through vesicles. Several ER chaperone proteins, including protein disulfide isomerase, correct the folding of newly made proteins. Our article summarizes the role of ER stress in DN.

\section{ER Stress}

The ER is committed to protein folding, maturation, quality control, and trafficking. The ER becomes stressed (ER stress) because of the accumulation of newly synthesized unfolded proteins. Moderate ER stress promotes the stability and recovery of the intracellular environment. Persistent ER stress slows protein folding, contributing to the accumulation of misfolded and unfolded proteins. Thus, ER stress ultimately leads to apoptosis, protein degradation, translation attenuation, and an antioxidant response.

ER stress can be divided into three types: the unfolded protein response (UPR), the ER overload response (EOR), and sterol regulatory element-binding protein (SREBP). The UPR and EOR are attributed to disordered protein processing. SREBP results from the depletion of newly synthesized sterol in the ER.

2.1. UPR. The UPR is activated by the accumulation of unfolded proteins and increases protein folding capacity [5, 6]. The UPR is regulated by three sensors to restore the normal function of the ER: protein-kinase-RNA-like ER kinase (PERK), inositol-requiring enzyme 1 (IRE-1), and activating transcription factor 6 (ATF6).

2.1.1. PERK. PERK is a transmembrane protein in the ER [7, 8]. Under normal conditions, PERK is inactivated by binding to glucose-regulated protein 78 (GRP78). Under pathological circumstances (such as hypoxia, ischemia, and oxidative stress), PERK is activated as a homodimer by dissociating 
from GRP78. Phosphorylated PERK phosphorylates eukaryotic translation initiation factor (eIF2), which attenuates mRNA translation. Additionally, phosphorylated PERK induces the transcription factors ATF4 and C/EBP homologous protein $(\mathrm{CHOP})$, which regulate the expression of genes involved in maintaining homeostasis [9]. Activated PERK also increases the expression of the transcription factor NRF2, which triggers an antioxidant response $[10,11]$.

2.1.2. IRE-1. IRE-1 is highly conserved in the ER. Interestingly, this protein is a kinase and an endoribonuclease. The RNase activity of IRE-1 increases the degradation of RNA and subsequently reduces protein synthesis. Previously, IRE-1 was shown to regulate cell survival and apoptosis [12]. Recent studies have changed this concept by demonstrating that IRE-1 directs UPR signaling and cell fate [13]. $\mathrm{X}$-box binding protein 1 (Xbp-1) was identified as a target of IRE-1 [14]. To enhance protein folding abilities, IRE-1 induces the mRNA expression of Xbp-1 and subsequently augments the transcription of ER quality-control components.

It has been proposed that IRE-1 can regulate apoptosis during ER stress. Repression of IRE-1 potentiates cell apoptosis by upregulating caspase-2 (Casp2). Additionally, activation of IRE-1-Casp2 events triggers cell death in the apoptotic phase.

2.1.3. ATF6. As the third sensor of the UPR, ATF6 is a transmembrane protein and transcription factor. Under conditions of non-ER stress, ATF6 is constitutively located in the ER and bound to GRP78 [15]. Under conditions of ER stress, ATF6 can translocate to the Golgi, where it is cleaved following GRP78 dissociation. Cleaved ATF6 can translocate to the nucleus and trigger Xbp-1 gene expression, providing an additional substrate for IRE-1 $[16,17]$. Some UPR proteins (such as ER chaperones) are direct targets of cleaved ATF6, which enhances ER protein folding capacity.

In summary, the UPR has evolved into an interconnected, dynamic, and flexible network of tubular and planar membranes. Under conditions of ER stress, the UPR attenuates the unfolded protein load in the ER. If successful in decreasing the number of misfolded proteins, the UPR is alleviated, and the cell survives. If ER stress persists and cannot be restored, the UPR induces apoptosis. Significantly, IRE-1 is necessary and sufficient to induce apoptosis, while PERK and AFT6 are dispensable in apoptosis. In certain cases, different sensors of ER stress might act as major executioners of cell death.

2.2. EOR. In contrast to the UPR, the EOR is characterized by the release of $\mathrm{Ca}^{2+}$ from the ER lumen to trigger the expression of reactive oxygen species (ROS) $[18,19]$. The enhanced ROS production activates NF- $\kappa \mathrm{B}$, namely, the EOR-Ca ${ }^{2+}$ ROS-NF- $\kappa$ B pathway. NF- $\kappa$ B is a transcription factor that regulates the expression of many genes involved in cell survival and cell proliferation. Interestingly, the EOR-Ca ${ }^{2+}$ ROS axis regulates cell death associated with oxidative stress and apoptosis, while the EOR-Ca ${ }^{2+}-\mathrm{ROS}-\mathrm{NF}-\kappa \mathrm{B}$ axis mediates cell viability due to the effects of NF- $\kappa$ B. The downstream target of NF- $\kappa \mathrm{B}$ has antiapoptotic effects. Thus, ROS-induced NF- $\kappa \mathrm{B}$ alleviates the detrimental effects of ROS.

In summary, the EOR-Ca ${ }^{2+}$-ROS pathway has two opposing effects on cell viability: the EOR-Ca ${ }^{2+}$-ROS pathway enhances apoptosis, while the ROS pathway activates NF- $\kappa \mathrm{B}$ and promotes cell viability [20].

2.3. SREBP. Intracellular cholesterol depletion leads to ER stress-induced SREBP activation [21]. Several publications have also demonstrated that ER stress is related to activated SREBP and lipid disorders [22-24]. Mammalian cells express three SREBP isoforms: SREBP-1a, SREBP-1c, and SREBP-2. SREBP-1a and SREBP-1c were reported to be associated with the biosynthesis of cholesterol and fatty acids. SREBP-2 is a more selective activator of cholesterol biosynthesis.

Current studies suggest three potential mechanisms of ER stress-induced activation of SREBP [25-27]: caspaseinduced SREBP cleavage, eIF2 phosphorylation-dependent downregulation of insulin-induced gene-1 and 2 (INSIG), and GRP78 dissociation from the SREBP cleavageactivation protein (SCAP)-SREBP complex.

Overall, ER stress is the first attempt to regulate protein folding demands, ER overload, and intracellular cholesterol depletion to restore homeostasis and functions to keep the cell alive and restore cellular functions. Each ER stress signal has unique and distinct targets, which act as homeostatic feedback loops to control ER stress [28]. If successful in restoring homeostasis, ER stress is alleviated, and the cell survives. However, if adaptive responses are lost, ER stress continues, leading to high or chronic ER stress. Unalleviated ER stress promotes cell death.

\section{Mechanism of ER Stress in DN}

$\mathrm{DN}$ is the leading cause of morbidity and end-stage renal disease. Hyperglycemia induces ER stress and apoptosis in renal cells [29]. Accumulating evidence has demonstrated that ER stress plays a substantial role in the development and pathogenesis of DN. The exact mechanisms are complex, have not been fully elucidated, and are summarized as follows:

3.1. Renal Epidermal Growth Factor Receptors (EGFRs). Previous studies have reported that EGFRs are activated in DN. Zhang et al. [30] demonstrated that inhibiting renal EGFRs with erlotinib reduced kidney ER stress and alleviated nephropathic changes in STZ mice, a finding likely associated with the inhibition of mTOR and activation of the AMPK pathway. Xu et al. [31] suggested that EGFR plays an essential role in the development of DN by enhancing ROS production and ER stress. Inhibiting EGFR alleviated renal damage via the EGFR/AKT/ROS/ER stress signaling pathway. Thus, direct inhibition of EGFR activity and/or inhibition of signaling pathways activated by EGFRs might be novel strategies to prevent and treat progressive renal damage in DN.

3.2. Hyperglycemia. Absolute or relative insulin deficiency leads to elevated blood glucose levels, termed hyperglycemia. Chronic high glucose (HG) exposure activates IRE1 to splice Xbp-1 mRNA, whereas acute exposure to HG activates IRE 
without Xbp-1 splicing [32]. Additionally, the activation of UPR pathways is amplified by supplementation with HG and FFAs [33]. HG stimulates renal proximal tubular cells and increases the accumulation of ROS, leading to enhanced ER stress. Several studies have shown HG-induced ER stress in vivo and in vitro $[34,35]$. Improving glucose metabolism might highlight one of the molecular mechanisms for clinical DN treatment in the future.

3.3. Reactive Oxygen Species. ROS play dual roles in ER stress [36]. On the one hand, ROS act as signaling intermediates that report ER stress to the UPR. Consequently, ER stress can be mitigated. On the other hand, when ER stress is not relieved over time, the delayed expression of proteins such as CHOP initiates a secondary increase in ROS. Additionally, the induction of ER oxidase 1 and calcium transfer across specialized ER-mitochondria leads to a secondary increase in ROS, contributing to cell death. The complexities of how ROS are formed and contribute to both homeostatic signaling and cell death raise numerous challenges in translating recent findings into clinical applications.

3.4. Angiotensin II Receptor Pathway. It is widely accepted that activation of the renin-angiotensin system (RAS), particularly the intrarenal RAS, plays a significant role in the pathophysiology of DN. Angiotensin II (Ang II), a profibrotic and proinflammatory peptide, is the major factor of the RAS. Angiotensin II (Ang II) plays a negative role in ER stressinduced apoptosis. Sun et al. [37] suggested that angiotensin-converting enzyme inhibitors (ACEIs) decrease ER stress-induced renal apoptosis in animal models of diabetes. Additionally, Ang II is associated with increased expression of ER chaperones and GRP78 (an ER stress marker) [38].

3.5. Free Fatty Acids (FFAs). Dietary fats and changes in lipid metabolism due to diabetes could contribute to increased FFA concentrations [39]. FFAs play a significant role in the pathogenesis of DN [40,41]. Saturated FFAs such as palmitic acid are proapoptotic factors in $\beta$-cells [42], while monounsaturated FFAs such as palmitoleic and oleic acid are capable of preventing/alleviating palmitic acid-induced apoptosis in pancreatic $\beta$-cells [43]. Palmitic acid induces ER stress in podocytes, leading to an unfolded protein response. Additionally, Sieber et al. [44] showed that monounsaturated palmitoleic acid and oleic acid decrease palmitic acid-induced cell death. Dietary shifting of the FFA balance toward unsaturated FFAs can delay the progression of DN.

3.6. Advanced Glycation End Products (AGEs). The formation of AGEs is associated with hyperglycemia. Emerging studies $[45,46]$ have indicated that the accumulation of AGEs induces podocyte apoptosis through ER stress, leading to albuminuria and renal damage [44, 47]. Additionally, AGEs induce enhanced GRP78 expression [48], which can be alleviated by TUDCA (an ER stress inhibitor mentioned previously) [49]. Thus, AGEs trigger ER stress in DN.

3.7. X-Box Binding Protein 1 (Xbp-1). Xbp-1 is a downstream transcription factor stimulated by ER stress that is spliced by activated IRE1 $\alpha$ [50, 51]. Increased spliced Xbp-1 (sXbp-1) reverses HG-induced reactive oxygen species production and extracellular matrix expression [29]. However, knocking down intrinsic sXbp-1 expression induces opposite effects. These findings suggest that the sXbp-1 pathway in ER stress plays a significant role in HG-induced oxidative stress and extracellular matrix synthesis [52].

3.8. Autophagy. Autophagy plays multiple roles in cells, such as those in cell growth, differentiation, and death [53]. The interaction of ER stress and autophagy in DN has been studied [54-56]. The basal level of autophagy in podocytes was reduced in an animal model of streptozotocin-induced diabetes. Similarly, the levels of autophagy markers were decreased in cultured glomerular epithelial cells (GECs) exposed to HG. The permeability of GECs was damaged by HG levels and alleviated by stimulating autophagy using rapamycin. Interestingly, TUDCA and salubrinal (both of which are ER stress inhibitors) attenuated HG-induced autophagy suppression.

\section{Roles of ER Stress in Various Renal Cells in DN}

Recent studies have demonstrated that ER stress is closely related to podocyte injury, glomerular endothelial cells (GECs), mesangial cells (MCs), and tubular epithelial cells. In patients with DN, hyperglycemia can induce ER stress in various ways, leading to cellular injury.

4.1. Podocytes. Podocyte injury is vital in the progression of DN. Emerging evidence suggests that ER stress is stimulated in patients with DN. Podocytes are likely susceptible to ER stress because of their large ER capacity and high levels of anabolic or catabolic activities.

AGEs and HG can trigger ER stress and apoptosis in podocytes that can be alleviated by ER stress suppressors. Tian et al. [57] suggested that emodin ameliorates renal damage in DN mice. Emodin decreases HG-induced ER stress in podocytes by counteracting the upregulation of phosphorylated PERK, phosphorylated eIF2 $\alpha$, ATF4, and CHOP [57]. Sieber et al. [44] showed that palmitic acid induces ER stress in podocytes and that palmitoleic acid and oleic acid alleviate the palmitic acid-induced UPR and prevent podocyte death. Yu et al. [58] suggested that Ang II induces podocyte foot process fusion and apoptosis via ER stress, effects that can be attenuated by curcumin.

Fan et al. [59] demonstrated that reticulon 1A (RTN1A) might be a key regulator of ER stress in podocytes in DN. In $\mathrm{db} / \mathrm{db}$ mice with unilateral nephrectomy (an animal model of progressive DN), RTN1A expression and ER stress were increased. In cultured podocytes, RTN1A mediates albumin-induced ER stress and apoptosis. The positive feedback loop between RTN1A and CHOP contributes to enhanced ER stress in podocytes.

Crosstalk between mTOR signaling and ER stress in podocytes has emerged recently. Lei et al. [60] demonstrated that HG induces podocyte injury through activated mTORinduced ER stress and apoptosis. 
Collectively, ER-induced podocyte injury is crucial for DN. Several stimulators (such as HG, AGEs, FFAs, and Ang II) induce ER stress in podocytes. RTN1A and mTOR might participate in these processes. Sustained ER stress leads to podocyte apoptosis and ultimately cell death. Indeed, the stimulators and mechanisms are largely unclear and require further study.

4.2. MCs. MCs are the principal components of the glomerular mesangium. MC injury leads to renal dysfunction, contributing to DN. Previous studies have demonstrated that ER stress induces MC injury in DN, which can be summarized as follows:

Hyperglycemia and ROS are widely recognized to stimulate ER stress in cultured MCs. Yao et al. [61] investigated the mechanism of HG-induced ER stress in MCs and found that HG induces ER stress through fatty acid-binding protein 4 (FABP4), a carrier protein for fatty acids. Hyperglycemia increases ROS in DN. Previous studies have demonstrated that increased ROS induce ER stress, leading to cell apoptosis. $\mathrm{Xu}$ et al. [31] suggested that the EGFR-AKT-ROS-ER stress pathway is present in STZ-induced diabetic mice and HG-treated MCs, and blocking EGFR might be a therapeutic strategy for DN.

The expression of profibrotic transforming growth factor- $\beta 1$ (TGF- $\beta 1$ ) is increased in MCs in diabetes. Several factors (such as And II and $\mathrm{HG}$ ) associated with DN can increase the expression of TGF- $\beta 1$ in MCs in vivo and in vitro [62-66]. Xu et al. [67] found that TGF- $\beta 1$ induces ER stress in MCs, an effect that can be augmented by Eprostanoid 2 receptor (EP2) deficiency.

Lipid-mediated ER stress in MCs was also studied. Park et al. [68] cultured rat MCs with palmitate, which mimics lipotoxicity, to determine the mechanism of lipotoxicityinduced mesangial cell injury and pathogenesis of DN. Researchers found that lipotoxicity induced ER stress via the protein arginine methyltransferase 1 (PRMT1), which exacerbated MC apoptosis. Interestingly, Yang et al. [69] proved that inflammation accelerates lipid-induced $\mathrm{MC}$ injury through ER stress. Hence, strategies to mediate the expression of PRMT1 can be applied to prevent or limit DN.

Asymmetric dimethylarginine- (ADMA-) induced ER stress in DN should be mentioned. Increased renal ADMA levels have been reported in DN [70]. Park et al. [71] demonstrated that elevated ADMA levels induce ER stress, leading to mitochondrial membrane potential injury and apoptosis in MCs.

In summary, hyperglycemia, ROS, TGF- $\beta 1$, and ADMA can induce ER stress in MCs. Sustained ER stress leads to $\mathrm{MC}$ apoptosis, which contributes to renal damage in DN.

4.3. ECs. Accumulating evidence supports that glomerular endothelial cells (GEnCs) are principally involved in the process of DN. However, the relationship between ER stress and GEnCs remains unclear.

Bi et al. [72] suggested that Ang II induces ER stress, leading to GEnC injury and accelerated renal damage. Angiopoietin 1 (Angpt1), a member of the angiopoietin family of growth factors, could attenuate these changes through the Tie2 receptor-ERK1/2-p38 MAPK pathways.

As mentioned previously, the serum concentration of ADMA increases early, even when the glomerular filtration rate (GFR) is still within the normal range in chronic kidney disease [73]. Guo et al. [74] suggested that ADMA promotes ER stress and GEnC apoptosis. Quercetin, one of the most important flavonoids, has been shown to attenuate ADMAinduced ER stress in GEnCs.

Advanced oxidation protein products (AOPPs) have been indicated to contribute to the development of DN. Liang et al. [75] pointed out that AOPPs induced ER stress in GEnCs by increasing the expression of GRP78 and CHOP, contributing to endothelial-to-mesenchymal transition.

To date, only a few reports have focused on the roles of ER stress in GEnCs in DN. Further studies are needed to improve the understanding of the exact mechanism of ER stress in GEnCs.

4.4. Tubular Epithelial Cells. Renal tubular epithelial cells (RTECs) are the key target cells that are highly vulnerable to damage in the context of diabetes and play a significant role in the development of DN. ER stress in RTECs is considered to contribute to renal injury in DN.

Previous studies have proven that $\mathrm{HG}$ and albumin induce ER stress in TECs. Shibusawa et al. [76] observed that HG induces ER stress through the eIF2 $\alpha$-ATF4-CHOP pathway in HK2 cells (a proximal tubular cell line). Dapagliflozin attenuates HG-induced ER stress in vivo and in vitro. Sun et al. used the rat RTEC line NRK-52E to investigate HGinduced ER stress, revealing that the histone deacetylase inhibitor valproic acid alleviates HG-induced ER stress. Jia et al. [77] examined albumin-induced ER stress in HK2 cells and found that albumin stimulated miR-4756 expression in HK2 cells. Furthermore, overexpression of miR-4756 enhanced albumin-induced ER stress by targeting Sestrin2 in HK2 cells. These observations were further supported by Lindenmeyer et al. [29], who investigated proteinuria- and hyperglycemia-induced ER stress in renal biopsies from patients with DN and cultured renal epithelial cells. The researchers pointed out that HG and albumin induced ER stress by producing free radicals, aberrant protein glycosylation, or increased membrane and protein turnover.

Urotensin II (UII) and its receptor are highly expressed in renal tissue in patients with DN [78]. Pang et al. [79] observed increased UII expression and ER stress in DN patients and diabetic mice, and UII induced epithelial-tomesenchymal transition (EMT) via the ER stress pathway in cultured HK2 cells. Additionally, a UII receptor antagonist and 4-PBA inhibited UII-induced ER stress and EMT.

PRMT1, which induces ER stress in the MCs, is upregulated in the serum of DN patients and triggers ER stress by activating PERK and ATF6 in HK2 cells [34]. Additionally, receptors or AGEs regulate ER stress in RTECs, which induce premature senescence through p21 signaling in DN [46]. Palmitic acid (PA) was also observed to activate ER stress in proximal tubular cells [80].

Overall, ER stress-induced tubular injury is significant in $\mathrm{DN}$. 


\section{Targeting ER Stress in DN}

Substantial progress has been achieved in treatments that target ER stress in DN. Food and Drug Administration- (FDA-) approved chemical chaperones, such as 4-PBA and TUDCA, are classic ER stress suppressors that enhance ER folding capacity, restore glucose tolerance, and improve insulin sensitivity in metabolic disorders associated with diabetes.

Several studies have demonstrated the therapeutic effects of herbal or plant medicines in the treatment of DN through targeting ER stress.

(1) Astragaloside IV: astragaloside IV (AS-IV) is a saponin and active component in Astragalus membranaceus Bge, and AS-IV exerts protective effects against renal ER stress in vivo and in vitro [81, 82]. Subsequently, Guo et al. [83] found that AS-IV alleviates ER stress-induced podocyte injury by upregulating sarco-ER $\mathrm{Ca}^{2+}$-ATPase 2 (SERCA2) expression in STZ-induced DN rats

(2) Huangkui: Huangkui is a traditional Chinese medicine that was approved by the China State FDA (z19990040) to treat nephritis. Ge et al. [84] demonstrated that Huangkui could improve renal damage by alleviating ER stress in DN rats

(3) Oleanolic acid (OA): OA naturally occurs in fruits and vegetables. Several studies $[85,86]$ have reported that OA has antioxidant, antiglycation, anti-inflammatory, and microbicidal activities. Additionally, OA has therapeutic effects on DN via ER stress reduction [87]

(4) Quercetin: quercetin is a potent antioxidant, and its function has been extensively studied because of its widespread distribution in various foods. Previous studies have demonstrated that quercetin exerts pharmacological effects, such as inhibiting platelet aggregation, hypertension, and lipid peroxidation [88-91]. In particular, in recent years, quercetin was found to improve diabetes-induced endothelial dysfunction by suppressing the ER stress pathway $[74,92]$

(5) Arctigenin: arctigenin (ATG) is a natural lignan compound mainly derived from the seeds of Arctium lappa. ATG has several bioactivities, such as antiinflammatory, antioxidative, and antidiabetic activities. Additionally, ATG has been demonstrated to be an ER stress inhibitor that suppresses the renal UPR in diabetic $\mathrm{db} / \mathrm{db}$ mice and cultured HK2 cells [93]

Additionally, several Western medicines can inhibit ER stress in DN.

(1) Aliskiren and valsartan: overactivation of the RAS plays significant roles in the progression of $\mathrm{DN}$. Drugs targeting the RAS, including angiotensinconverting enzyme inhibitors (ACEIs) and Ang II

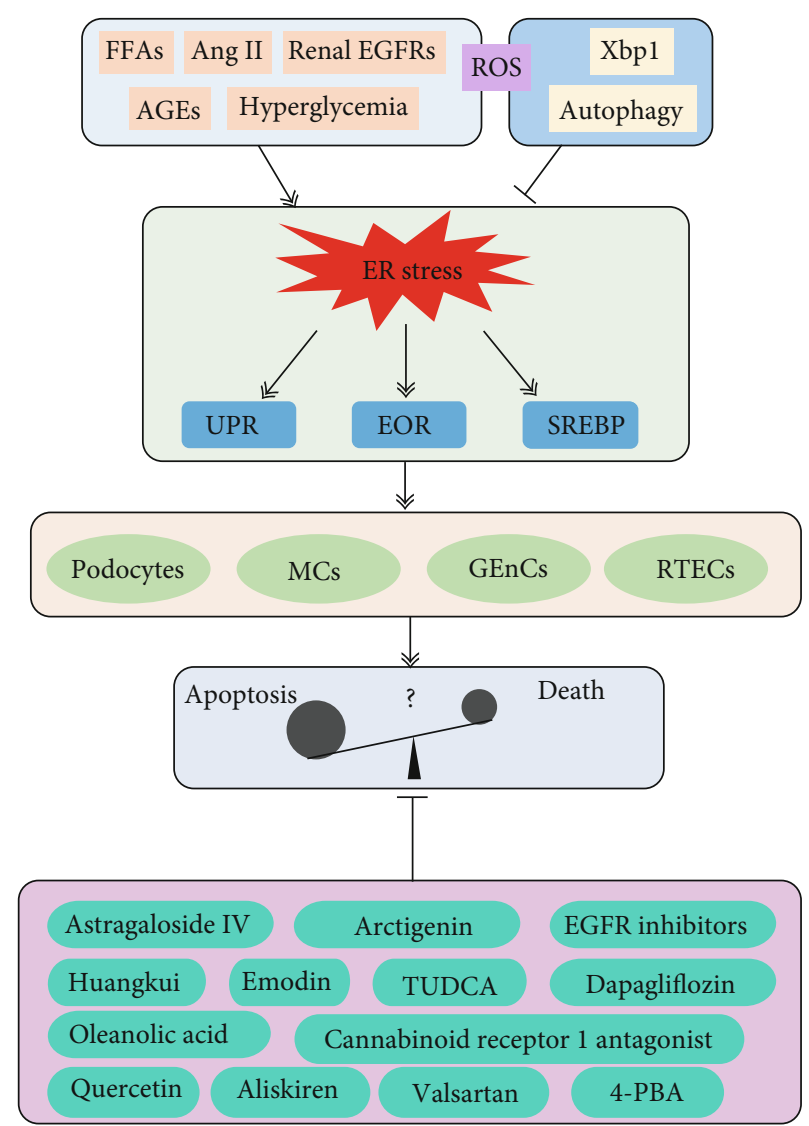

FIgURE 1: Role of endoplasmic reticulum (ER) stress in diabetic nephropathy. In diabetes mellitus, various effectors, including FFAs, Ang II, renal EGFRs, AGEs, and hyperglycemia, contribute to ER stress. Xbp-1 and autophagy can inhibit ER stress. The ROS had dual effects on ER stress. ER stress can be divided into three types: the UPR, the EOR, and SREBP. ER stress is closely related to podocyte injury, glomerular endothelial cells (GECs), mesangial cells (MCs), and renal tubular epithelial cells (RTECs). Several drugs have been demonstrated to inhibit ER stress in DN.

type I receptor blockers (ARBs), have been used to cure DN. Aliskiren (an ACEI) and valsartan (an ARB) inhibit ER stress. Dual treatment with aliskiren and valsartan induces additive therapeutic effects in the treatment of DN [94]

(2) Cannabinoid receptor 1 antagonist: cannabinoid receptor $1\left(\mathrm{CB}_{1} \mathrm{R}\right)$ is highly expressed in the kidneys of diabetic mice. $\mathrm{CB}_{1}$ inhibition prevents diabetesinduced renal damage. CB1 mediates HG- [95] or palmitic acid-induced ER stress/apoptosis [96] in cultured rat MCs and human RTECs

(3) Chemical chaperones: chemical chaperones are small molecules that stabilize protein conformation, improve the ER folding capacity, and facilitate the trafficking of mutant proteins. These compounds include tauroursodeoxycholic acid (TUDCA) and 4phenylbutyrate (4-PBA)

TUDCA is a secondary bile acid [97] that has been proven to have beneficial effects on various diseases, such as 


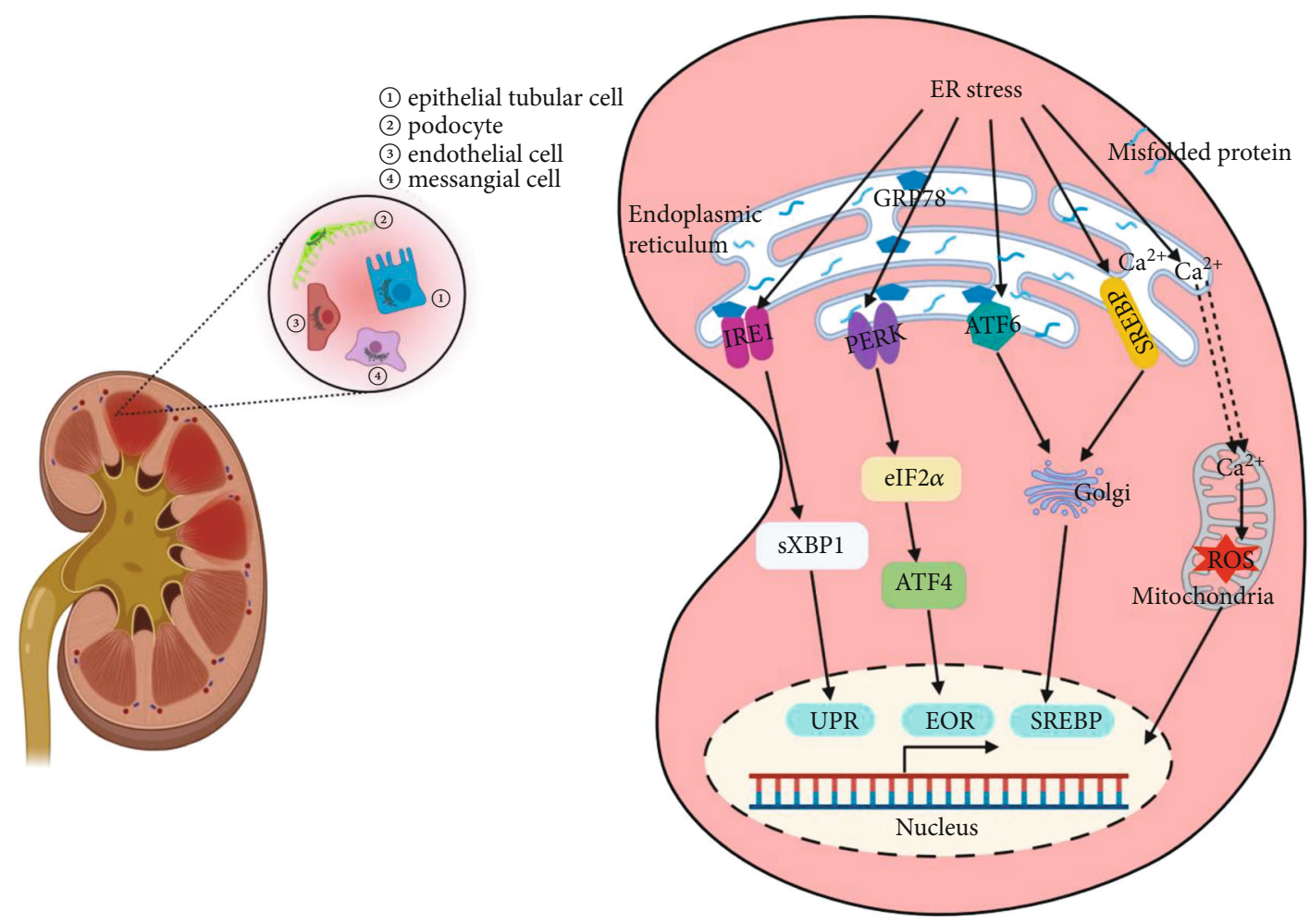

FIGURE 2: Endoplasmic reticulum (ER) stress in various renal cells in diabetic nephropathy (DN). ER stress participates in the development and pathogenesis of DN. When the ER is stressed, for example, by misfolded proteins or impaired $\mathrm{Ca}^{2+}$ homeostasis, the UPR, EOR, or SREBP is initiated. ER stress is mainly located in podocytes, glomerular endothelial cells, mesangial cells, and renal tubular epithelial cells in DN.

diabetes, obesity, and neurodegenerative diseases. The mechanism of these cytoprotective activities is largely due to the alleviation of ER stress.

4-PBA is a low-molecular-weight chemical chaperone that is widely used for urea cycle disorders. It has been demonstrated that 4-PBA can suppress ER stress, leading to the normalization of hyperglycemia and insulin resistance [98100]. Cao et al. [101] suggested that 4-PBA prevents ER stress-induced podocyte apoptosis in type 2 diabetic mice. Additionally, three widely used ER stress inducers, tunicamycin, DTT, and MG132, were applied to evaluate ER stress in animals and cell lines. 4-PBA rescued drug-induced ER stress [102].

Additionally, some agents targeting pathways that exhibit remarkable functions can modify ER stress. GSK2606414 (inhibition of PERK), MKC-3946 (inhibition of IRE1 $\alpha$ ), salubrinal (inhibition of eIF2 $\alpha$ phosphatases), and trazodone (inhibition of ATF4 induction) are widely used to attenuate ER stress in vivo and in vitro.

Finally, some old drugs exert novel effects in inhibiting ER stress in DN and were used only in research phases. Emodin, EGFR inhibitors, and dapagliflozin have been found to exert potential effects on ER stress and improve renal function in vitro and in animal studies. Furthermore, Shih et al. found that dapagliflozin treatment suppresses myocardial ER stress in patients with DN [103].

In summary, attention should be given to the treatment of ER stress. First, the abovementioned drugs may have multiple biological effects, indicating that these drugs are not spe- cific in suppressing ER stress. Hence, the development of new medications that target ER stress is urgently needed. Additionally, the ER stress response can be a double-edged sword. Moderate ER stress restores the intracellular environment, while sustained ER stress contributes to renal damage in DN. Focused studies on ER stress in humans are encouraged [104-106]. Thus, a better understanding of the mechanism of this process would be helpful to improve therapies for DN.

\section{Conclusion and Perspectives}

ER stress can be induced by several stimuli that alter homeostatic cellular functions (Figure 1). Increasing evidence suggests that ER stress plays important roles in the development and progression of DN.

Our understanding of the mechanism of acute and chronic ER stress in DN is rapidly increasing. ER stress clearly has both useful and harmful effects on the kidney. ER stress can recover normal organ function. However, chronic activation of ER stress can lead to chronic renal damage, which is associated with chronic renal failure. The upregulation of ER stress (by one or two ER stress markers) should not be interpreted as harmful. Additionally, some questions should also be resolved: Does activation of one pathway constitute the ER stress response, or must all three sensors (PERK, IRE-1, and ATF6) of the UPR be activated to be considered a true ER stress response? Do specific inducers of the UPR activate all three arms of the UPR? 
In this review, we summarized the roles of ER stress in different kidney cell types in DN (Figure 2). Many questions persist, mainly concerning which renal cells are the most affected by ER stress and whether all cells are affected similarly. These questions deserve further research. Additionally, evaluation of the temporal patterns of ER stress activation was neglected. Timing seems to play an important role in outcomes.

An improved understanding of the mechanisms of ER stress will lead to improved therapeutic strategies targeting ER stress as a treatment for DN. Considering the protective effects of ER stress, the blind pursuit of ER stress inhibition might undoubtedly be a mistake. Considering the adverse effects of ER stress, the development of new medications that can target ER stress in a cell- and disease-specific manner is urgently needed. Therapies targeting ER stress have shown increased potential and bright prospects. Focused studies on ER stress in humans would increase our understanding of targeted DN therapies.

In summary, ongoing research is required to solve these problems. Finally, the development and knowledge gained would similarly help to promote the treatment of both DN and ER stress-related disorders.

\section{Data Availability}

The data used to support the findings of this study are available from the corresponding author upon request.

\section{Conflicts of Interest}

The authors declare no conflicts of interests.

\section{Authors' Contributions}

Lihua Ni and Cheng Yuan contributed equally to this work.

\section{Acknowledgments}

This work was supported by grants from the Fundamental Research Funds for the Central Universities (2042021kf0150 and 2042020kf0137), the Zhongnan Hospital of Wuhan University Science, Technology and Innovation Seed Fund Project (cxpy2020027 and znpy2019036), the Excellent Doctor (Post), Zhongnan Hospital of Wuhan University (ZNYB2020009), the Hubei Province Health and Family Planning Scientific Research Project (WJ2019MB103), the Zhongnan Hospital of Wuhan University Science, Technology and Innovation Seed Fund (znpy2017044), the Clinical Research Project for Wu Jieping Medical Foundation (320.6750.19089-58), and the Research Fund from Medical Sci-Tech Innovation Platform of Zhongnan Hospital, Wuhan University (PTXM2020028).

\section{References}

[1] S. Xiong, Y. Han, P. Gao, H. Zhao, N. Jiang, and L. Sun, "AdipoRon protects against tubular injury in diabetic nephropathy by inhibiting endoplasmic reticulum stress," Oxidative
Medicine and Cellular Longevity, vol. 2020, Article ID 6104375, 15 pages, 2020.

[2] H. Liu and H. L. Sun, "LncRNA TCF7 triggered endoplasmic reticulum stress through a sponge action with mi R-200c in patients with diabetic nephropathy," European Review for Medical and Pharmacological Sciences, vol. 23, no. 13, pp. 5912-5922, 2019.

[3] J. Zhang, X. J. Dong, M. R. Ding et al., "Resveratrol decreases high glucose-induced apoptosis in renal tubular cells via suppressing endoplasmic reticulum stress," Molecular Medicine Reports, vol. 22, no. 5, pp. 4367-4375, 2020.

[4] V. Choudhary and R. Schneiter, "A unique junctional interface at contact sites between the endoplasmic reticulum and lipid droplets," Frontiers in Cell and Development Biology, vol. 9, p. 650186, 2021.

[5] R. Ghemrawi and M. Khair, "Endoplasmic reticulum stress and unfolded protein response in neurodegenerative diseases," International journal of molecular sciences, vol. 21, no. 17 , p. $6127,2020$.

[6] D. Stöhr, A. Jeltsch, and M. Rehm, “TRAIL receptor signaling: from the basics of canonical signal transduction toward its entanglement with ER stress and the unfolded protein response," International Review of Cell and Molecular Biology, vol. 351, pp. 57-99, 2020.

[7] M. Schröder and R. J. Kaufman, "Divergent roles of IRE1alpha and PERK in the unfolded protein response," Current Molecular Medicine, vol. 6, no. 1, pp. 5-36, 2006.

[8] W. Rozpedek, L. Markiewicz, J. Alan Diehl, D. Pytel, and I. Majsterek, "Unfolded protein response and PERK kinase as a new therapeutic target in the pathogenesis of Alzheimer's disease," Current Medicinal Chemistry, vol. 22, no. 27, pp. 3169-3184, 2015.

[9] J. Guo, R. Ren, K. Sun, J. He, and J. Shao, "PERK signaling pathway in bone metabolism: friend or foe?," Cell Proliferation, vol. 54, no. 4, article e13011, 2021.

[10] W. Huang, C. C. Liao, Y. Han et al., "Co-activation of Akt, Nrf2, and NF- $\kappa$ B signals under UPR(ER) in torpid Myotis ricketti bats for survival," Communications biology, vol. 3, no. 1, p. 658, 2020.

[11] Y. Xu, H. Yuan, Y. Luo, Y.-J. Zhao, and J.-H. Xiao, "Ganoderic acid D protects human amniotic mesenchymal stem cells against oxidative stress-induced senescence through the PERK/NRF2 signaling pathway," Oxidative Medicine and Cellular Longevity, vol. 2020, Article ID 8291413, 18 pages, 2020.

[12] N. Siwecka, W. Rozpędek-Kamińska, A. Wawrzynkiewicz, D. Pytel, J. A. Diehl, and I. Majsterek, "The Structure, Activation and signaling of IRE1 and its role in determining cell fate," Biomedicine, vol. 9, no. 2, 2021.

[13] S. R. Barez, A. M. Atar, and M. Aghaei, "Mechanism of inositol-requiring enzyme 1-alpha inhibition in endoplasmic reticulum stress and apoptosis in ovarian cancer cells," Journal of cell communication and signaling, vol. 14, no. 4, pp. 403-415, 2020.

[14] S. Wang, Z. Wang, Q. Fan et al., "Ginkgolide K protects the heart against endoplasmic reticulum stress injury by activating the inositol-requiring enzyme $1 \alpha / \mathrm{X}$ box-binding protein-1 pathway," British Journal of Pharmacology, vol. 173, no. 15, pp. 2402-2418, 2016.

[15] X. Wang, T. Zhang, H. Mao et al., "Grass carp ( _Ctenopharyngodon idella_ ) ATF6 (activating transcription factor 
6) modulates the transcriptional level of _GRP78_ and _GRP94_ in CIK cells," Fish \& Shellfish Immunology, vol. 52, pp. 65-73, 2016.

[16] R. B. Sharma, C. Darko, and L. C. Alonso, "Intersection of the ATF6 and XBP1 ER stress pathways in mouse islet cells," The Journal of Biological Chemistry, vol. 295, no. 41, pp. 1416414177, 2020.

[17] J. An, X. Zhang, K. Jia et al., "Trichostatin A increases BDNF protein expression by improving XBP-1s/ATF6/GRP78 axis in Schwann cells of diabetic peripheral neuropathy," Biomedicine \& Pharmacotherapy, vol. 133, p. 111062, 2021.

[18] S. Li, L. Ye, X. Yu et al., "Hepatitis C virus NS4B induces unfolded protein response and endoplasmic reticulum overload response-dependent NF- $\kappa \mathrm{B}$ activation," Virology, vol. 391, no. 2, pp. 257-264, 2009.

[19] G. Waris, A. Livolsi, V. Imbert, J. F. Peyron, and A. Siddiqui, "Hepatitis $\mathrm{C}$ virus NS5A and subgenomic replicon activate NF-kappaB via tyrosine phosphorylation of IkappaBalpha and its degradation by calpain protease," The Journal of Biological Chemistry, vol. 278, no. 42, pp. 40778-40787, 2003.

[20] L. Kong, S. Li, M. Huang et al., "The roles of endoplasmic reticulum overload response induced by HCV and NS4B protein in human hepatocyte viability and virus replication," PLoS One, vol. 10, no. 4, article e0123190, 2015.

[21] S. M. Colgan, A. A. Hashimi, and R. C. Austin, "Endoplasmic reticulum stress and lipid dysregulation," Expert Reviews in Molecular Medicine, vol. 13, article e4, 2011.

[22] L. Su, L. Zhou, F. J. Chen et al., "Cideb controls sterolregulated ER export of SREBP/SCAP by promoting cargo loading at ER exit sites," The EMBO Journal, vol. 38, no. 8, 2019.

[23] Y. R. Kim, E. J. Lee, K. O. Shin et al., "Hepatic triglyceride accumulation via endoplasmic reticulum stress-induced SREBP-1 activation is regulated by ceramide synthases," Experimental \& Molecular Medicine, vol. 51, no. 11, pp. 116, 2019.

[24] P. Singh, M. I. Reza, A. A. Syed et al., "PSTi8 with metformin ameliorates perimenopause induced steatohepatitis associated ER stress by regulating SIRT-1/SREBP-1c axis," Heliyon, vol. 6 , no. 12, article e05826, 2020.

[25] H. Wang, G. Kouri, and C. B. Wollheim, "ER stress and SREBP-1 activation are implicated in beta-cell glucolipotoxicity," Journal of Cell Science, vol. 118, Part 17, pp. 3905-3915, 2005.

[26] Y. R. Kim, E. J. Lee, K. O. Shin et al., “Deep proteome profiling of the hippocampus in the 5XFAD mouse model reveals biological process alterations and a novel biomarker of Alzheimer's disease," Experimental \& Molecular Medicine, vol. 51, no. 11, pp. 1-17, 2019.

[27] F. Damiano, S. Alemanno, G. V. Gnoni, and L. Siculella, "Translational control of the sterol-regulatory transcription factor SREBP-1 mRNA in response to serum starvation or ER stress is mediated by an internal ribosome entry site," The Biochemical Journal, vol. 429, no. 3, pp. 603-612, 2010.

[28] S. A. Oakes and F. R. Papa, "The role of endoplasmic reticulum stress in human pathology," Annual Review of Pathology, vol. 10, pp. 173-194, 2015.

[29] M. T. Lindenmeyer, M. P. Rastaldi, M. Ikehata et al., "Proteinuria and hyperglycemia induce endoplasmic reticulum stress," Journal of the American Society of Nephrology, vol. 19, no. 11, pp. 2225-2236, 2008.
[30] M. Z. Zhang, Y. Wang, P. Paueksakon, and R. C. Harris, "Epidermal growth factor receptor inhibition slows progression of diabetic nephropathy in association with a decrease in endoplasmic reticulum stress and an increase in autophagy," Diabetes, vol. 63, no. 6, pp. 2063-2072, 2014

[31] Z. Xu, Y. Zhao, P. Zhong et al., "EGFR inhibition attenuates diabetic nephropathy through decreasing ROS and endoplasmic reticulum stress," Oncotarget, vol. 8, no. 20, pp. $32655-$ 32667, 2017.

[32] K. L. Lipson, S. G. Fonseca, S. Ishigaki et al., "Regulation of insulin biosynthesis in pancreatic beta cells by an endoplasmic reticulum-resident protein kinase IRE1," Cell Metabolism, vol. 4, no. 3, pp. 245-254, 2006.

[33] S. H. Back and R. J. Kaufman, "Endoplasmic reticulum stress and type 2 diabetes," Annual Review of Biochemistry, vol. 81, pp. 767-793, 2012.

[34] Y. Y. Chen, X. F. Peng, G. Y. Liu et al., "Protein arginine methyltranferase-1 induces ER stress and epithelialmesenchymal transition in renal tubular epithelial cells and contributes to diabetic nephropathy," Biochimica et Biophysica Acta - Molecular Basis of Disease, vol. 1865, no. 10, pp. 2563-2575, 2019.

[35] X. M. Zhang, Y. Z. Wang, J. D. Tong et al., "Pyruvate alleviates high glucose-induced endoplasmic reticulum stress and apoptosis in HK-2 cells," FEBS Open Bio, vol. 10, no. 5, pp. 827-834, 2020.

[36] C. D. Ochoa, R. F. Wu, and L. S. Terada, "ROS signaling and ER stress in cardiovascular disease," Molecular Aspects of Medicine, vol. 63, pp. 18-29, 2018.

[37] H. L. Sun, L. Sun, Y. Y. Li et al., "ACE-inhibitor suppresses the apoptosis induced by endoplasmic reticulum stress in renal tubular in experimental diabetic rats," Experimental and Clinical Endocrinology \& Diabetes, vol. 117, no. 7, pp. 336-344, 2009.

[38] K. I. Okada, T. Minamino, Y. Tsukamoto et al., "Prolonged endoplasmic reticulum stress in hypertrophic and failing heart after aortic constriction: possible contribution of endoplasmic reticulum stress to cardiac myocyte apoptosis," Circulation, vol. 110, no. 6, pp. 705-712, 2004.

[39] S. ISA, A. C. Blindauer, and J. A. Stewart, "Changes in plasma free fatty acids associated with type-2 diabetes," Nutrients, vol. 11, no. 9, 2019

[40] J. L. Tao, Y. B. Wen, B. Y. Shi et al., "Endoplasmic reticulum stress is involved in podocyte apoptosis induced by saturated fatty acid palmitate," Chinese Medical Journal, vol. 125, no. 17, pp. 3137-3142, 2012.

[41] J. Sieber and A. W. Jehle, "Free fatty acids and their metabolism affect function and survival of podocytes," Frontiers in endocrinology, vol. 5, p. 186, 2014.

[42] K. Maedler, G. A. Spinas, D. Dyntar, W. Moritz, N. Kaiser, and M. Y. Donath, "Distinct effects of saturated and monounsaturated fatty acids on beta-cell turnover and function," Diabetes, vol. 50, no. 1, pp. 69-76, 2001.

[43] K. Maedler, J. Oberholzer, P. Bucher, G. A. Spinas, and M. Y. Donath, "Monounsaturated fatty acids prevent the deleterious effects of palmitate and high glucose on human pancreatic beta-cell turnover and function," Diabetes, vol. 52, no. 3, pp. 726-733, 2003.

[44] J. Sieber, M. T. Lindenmeyer, K. Kampe et al., "Regulation of podocyte survival and endoplasmic reticulum stress by fatty 
acids," American Journal of Physiology. Renal Physiology, vol. 299, no. 4, pp. F821-F829, 2010.

[45] R. Inagi, "Inhibitors of advanced glycation and endoplasmic reticulum stress," Methods in Enzymology, vol. 491, pp. 361-380, 2011.

[46] J. Liu, K. Huang, G. Y. Cai et al., "Receptor for advanced glycation end-products promotes premature senescence of proximal tubular epithelial cells via activation of endoplasmic reticulum stress-dependent p21 signaling," Cellular Signalling, vol. 26, no. 1, pp. 110-121, 2014.

[47] P. Y. Chuang, Q. Yu, W. Fang, J. Uribarri, and J. C. He, "Advanced glycation endproducts induce podocyte apoptosis by activation of the FOXO4 transcription factor," Kidney International, vol. 72, no. 8, pp. 965-976, 2007.

[48] U. Özcan, Q. Cao, E. Yilmaz et al., "Endoplasmic reticulum stress links obesity, insulin action, and type 2 diabetes," Science, vol. 306, no. 5695, pp. 457-461, 2004.

[49] Y. Chen, C. P. Liu, K. F. Xu et al., "Effect of taurineconjugated ursodeoxycholic acid on endoplasmic reticulum stress and apoptosis induced by advanced glycation end products in cultured mouse podocytes," American Journal of Nephrology, vol. 28, no. 6, pp. 1014-1022, 2008.

[50] C. M. Zhou, L. M. Luo, P. Lin et al., “Annexin A2 regulates unfolded protein response via IRE1-XBP1 axis in macrophages duringP. aeruginosainfection," Journal of Leukocyte Biology, 2020.

[51] W. Xu, C. Wang, and J. Hua, "X-box binding protein 1 (XBP1) function in diseases," Cell Biology International, vol. 45, no. 4, pp. 731-739, 2021.

[52] D. Shao, J. Liu, J. Ni et al., "Suppression of XBP1S mediates high glucose-induced oxidative stress and extracellular matrix synthesis in renal mesangial cell and kidney of diabetic rats," PLoS One, vol. 8, no. 2, article e56124, 2013.

[53] A. V. Cybulsky, "Endoplasmic reticulum stress, the unfolded protein response and autophagy in kidney diseases," Nature Reviews. Nephrology, vol. 13, no. 11, pp. 681-696, 2017.

[54] S. Kume, K. Yamahara, M. Yasuda, H. Maegawa, and D. Koya, "Autophagy: emerging therapeutic target for diabetic nephropathy," Seminars in Nephrology, vol. 34, no. 1, pp. 9-16, 2014.

[55] Y. Ding and M. E. Choi, "Autophagy in diabetic nephropathy," The Journal of Endocrinology, vol. 224, no. 1, pp. R15R30, 2015.

[56] O. Lenoir, P. L. Tharaux, and T. B. Huber, "Autophagy in kidney disease and aging: lessons from rodent models," Kidney International, vol. 90, no. 5, pp. 950-964, 2016.

[57] N. Tian, Y. Gao, X. Wang et al., "Emodin mitigates podocytes apoptosis induced by endoplasmic reticulum stress through the inhibition of the PERK pathway in diabetic nephropathy," Drug Design, Development and Therapy, vol. 12, pp. 2195-2211, 2018.

[58] N. Yu, L. Yang, L. Ling et al., "Curcumin attenuates angiotensin II-induced podocyte injury and apoptosis by inhibiting endoplasmic reticulum stress," FEBS Open Bio, vol. 10, no. 10, pp. 1957-1966, 2020.

[59] Y. Fan, J. Zhang, W. Xiao et al., "Rtn1a-mediated endoplasmic reticulum stress in podocyte injury and diabetic nephropathy," Scientific Reports, vol. 7, no. 1, p. 323, 2017.

[60] J. Lei, L. Zhao, Y. Zhang, Y. Wu, and Y. Liu, "High glucoseinduced podocyte injury involves activation of mammalian target of rapamycin (mTOR)-induced endoplasmic reticu- lum (ER) stress," Cellular Physiology and Biochemistry, vol. 45, no. 6, pp. 2431-2443, 2018.

[61] F. Yao, Z. Li, T. Ehara et al., "Fatty acid-binding protein 4 mediates apoptosis via endoplasmic reticulum stress in mesangial cells of diabetic nephropathy," Molecular and Cellular Endocrinology, vol. 411, pp. 232-242, 2015.

[62] K. Sharma and F. N. Ziyadeh, "Hyperglycemia and diabetic kidney disease. The case for transforming growth factorbeta as a key mediator," Diabetes, vol. 44, no. 10, pp. 11391146, 1995.

[63] F. N. Ziyadeh, B. B. Hoffman, D. C. Han et al., "Long-term prevention of renal insufficiency, excess matrix gene expression, and glomerular mesangial matrix expansion by treatment with monoclonal antitransforming growth factor-beta antibody in $\mathrm{db} / \mathrm{db}$ diabetic mice," Proceedings of the National Academy of Sciences of the United States of America, vol. 97, no. 14, pp. 8015-8020, 2000.

[64] T. Yamamoto, T. Nakamura, N. A. Noble, E. Ruoslahti, and W. A. Border, "Expression of transforming growth factor beta is elevated in human and experimental diabetic nephropathy," Proceedings of the National Academy of Sciences of the United States of America, vol. 90, no. 5, pp. 1814-1818, 1993.

[65] M. Kato and R. Natarajan, "Diabetic nephropathy-emerging epigenetic mechanisms," Nature Reviews. Nephrology, vol. 10, no. 9, pp. 517-530, 2014.

[66] K. Reidy, H. M. Kang, T. Hostetter, and K. Susztak, "Molecular mechanisms of diabetic kidney disease," The Journal of Clinical Investigation, vol. 124, no. 6, pp. 2333-2340, 2014.

[67] Y. Xu, J. Wang, T. Pan et al., "Role of the ER stress in prostaglandin E2/E-prostanoid 2 receptor involved TGF- $\beta 1$ induced mice mesangial cell injury," Molecular and Cellular Biochemistry, vol. 411, no. 1-2, pp. 43-55, 2016.

[68] M. J. Park, H. J. Han, and D. I. Kim, "Lipotoxicity-induced PRMT1 exacerbates mesangial cell apoptosis via endoplasmic reticulum stress," International Journal of Molecular Sciences, vol. 18, no. 7, 2017.

[69] H. Yang, J. Cui, J. Shi et al., "Endoplasmic reticulum stress participates in inflammation-accelerated, lipid-mediated injury of human glomerular mesangial cells," Nephrology (Carlton), vol. 22, no. 3, pp. 234-242, 2017.

[70] K. O. Hanai, T. Babazono, I. Nyumura et al., "Asymmetric dimethylarginine is closely associated with the development and progression of nephropathy in patients with type 2 diabetes," Nephrology, Dialysis, Transplantation, vol. 24, no. 6, pp. 1884-1888, 2009.

[71] M. J. Park, K. S. Oh, J. H. Nho, G. Y. Kim, and D. I. Kim, "Asymmetric dimethylarginine (ADMA) treatment induces apoptosis in cultured rat mesangial cells via endoplasmic reticulum stress activation," Cell Biology International, vol. 40, no. 6, pp. 662-670, 2016.

[72] X. Bi, J. Niu, W. Ding, M. Zhang, M. Yang, and Y. Gu, "Angiopoietin-1 attenuates angiotensin II-induced ER stress in glomerular endothelial cells via a Tie2 receptor/ERK1/2p38 MAPK-dependent mechanism," Molecular and Cellular Endocrinology, vol. 428, pp. 118-132, 2016.

[73] J. T. Kielstein, R. H. Böger, S. M. Bode-Böger et al., "Marked increase of asymmetric dimethylarginine in patients with incipient primary chronic renal disease," Journal of the American Society of Nephrology, vol. 13, no. 1, pp. 170176, 2002. 
[74] W. Guo, J. Ding, A. Zhang et al., "The inhibitory effect of quercetin on asymmetric dimethylarginine-induced apoptosis is mediated by the endoplasmic reticulum stress pathway in glomerular endothelial cells," International Journal of Molecular Sciences, vol. 15, no. 1, pp. 484-503, 2014.

[75] X. Liang, N. Duan, Y. Wang et al., "Advanced oxidation protein products induce endothelial-to-mesenchymal transition in human renal glomerular endothelial cells through induction of endoplasmic reticulum stress," Journal of Diabetes and its Complications, vol. 30, no. 4, pp. 573-579, 2016.

[76] R. Shibusawa, E. Yamada, S. Okada et al., "Dapagliflozin rescues endoplasmic reticulum stress-mediated cell death," Scientific Reports, vol. 9, no. 1, p. 9887, 2019.

[77] Y. Jia, Z. Zheng, Y. Yang et al., "miR-4756 promotes albumininduced renal tubular epithelial cell epithelial-tomesenchymal transition and endoplasmic reticulum stress via targeting Sestrin2," Journal of Cellular Physiology, vol. 234, no. 3, pp. 2905-2915, 2019.

[78] R. G. Langham, D. J. Kelly, R. M. Gow et al., "Increased expression of urotensin II and urotensin II receptor in human diabetic nephropathy," American Journal of Kidney Diseases, vol. 44, no. 5, pp. 826-831, 2004.

[79] X. X. Pang, Q. Bai, F. Wu, G. J. Chen, A. H. Zhang, and C. S. Tang, "Urotensin II induces ER stress and EMT and increase extracellular matrix production in renal tubular epithelial cell in early diabetic mice," Kidney \& Blood Pressure Research, vol. 41, no. 4, pp. 434-449, 2016.

[80] H. Sasaki, A. Kamijo-Ikemori, T. Sugaya et al., "Urinary fatty acids and liver-type fatty acid binding protein in diabetic nephropathy," Nephron. Clinical Practice, vol. 112, no. 3, pp. c148-c156, 2009.

[81] H. Guo, Y. Wang, X. Zhang et al., "Astragaloside IV protects against podocyte injury via SERCA2-dependent ER stress reduction and $\mathrm{AMPK} \alpha$-regulated autophagy induction in streptozotocin-induced diabetic nephropathy," Scientific Reports, vol. 7, no. 1, p. 6852, 2017.

[82] Y. Ju, Y. Su, Q. Chen et al., "Protective effects of astragaloside IV on endoplasmic reticulum stress-induced renal tubular epithelial cells apoptosis in type 2 diabetic nephropathy rats," Biomedicine \& Pharmacotherapy, vol. 109, pp. 84-92, 2019.

[83] H. Guo, A. Cao, S. Chu et al., "Astragaloside IV attenuates podocyte apoptosis mediated by endoplasmic reticulum stress through upregulating sarco/endoplasmic reticulum $\mathrm{Ca}(2+)$-ATPase 2 expression in diabetic nephropathy," Frontiers in Pharmacology, vol. 7, p. 500, 2016.

[84] J. Ge, J. J. Miao, X. Y. Sun, and J. Y. Yu, "Huangkui capsule, an extract from Abelmoschus manihot (L.) medic, improves diabetic nephropathy via activating peroxisome proliferatoractivated receptor (PPAR) $-\alpha / \gamma$ and attenuating endoplasmic reticulum stress in rats," Journal of Ethnopharmacology, vol. 189, pp. 238-249, 2016.

[85] N. Sultana and A. Ata, "Oleanolic acid and related derivatives as medicinally important compounds," Journal of Enzyme Inhibition and Medicinal Chemistry, vol. 23, no. 6, pp. 739756, 2008.

[86] D. Gao, Q. Li, Y. Li et al., "Antidiabetic potential of oleanolic acid from Ligustrum lucidum Ait," Canadian Journal of Physiology and Pharmacology, vol. 85, no. 11, pp. 10761083, 2007.

[87] E. S. Lee, H. M. Kim, J. S. Kang et al., "Oleanolic acid and Nacetylcysteine ameliorate diabetic nephropathy through reduction of oxidative stress and endoplasmic reticulum stress in a type 2 diabetic rat model," Nephrology, Dialysis, Transplantation, vol. 31, no. 3, pp. 391-400, 2016.

[88] W. F. Lai and W. T. Wong, "Design and optimization of quercetin-based functional foods," Critical Reviews in Food Science and Nutrition, pp. 1-17, 2021.

[89] J. C. Brito, W. G. Lima, L. P. Cordeiro, and W. S. da Cruz Nizer, "Effectiveness of supplementation with quercetintype flavonols for treatment of viral lower respiratory tract infections: systematic review and meta-analysis of preclinical studies," Phytotherapy Research, 2021.

[90] G. M. Albadrani, M. N. Binmowyna, M. N. Bin-Jumah, G. ElAkabawy, H. Aldera, and A. M. Al-Farga, "Quercetin protects against experimentally-induced myocardial infarction in rats by an antioxidant potential and concomitant activation of signal transducer and activator of transcription 3," Journal of Physiology and Pharmacology, vol. 71, no. 6, 2020.

[91] S. Dini, M. Zakeri, S. Ebrahimpour, F. Dehghanian, and A. Esmaeili, "Quercetin-conjugated superparamagnetic iron oxide nanoparticles modulate glucose metabolism-related genes and miR-29 family in the hippocampus of diabetic rats," Scientific Reports, vol. 11, no. 1, p. 8618, 2021.

[92] N. Suganya, S. Dornadula, S. Chatterjee, and R. K. Mohanram, "Quercetin improves endothelial function in diabetic rats through inhibition of endoplasmic reticulum stressmediated oxidative stress," European Journal of Pharmacology, vol. 819, pp. 80-88, 2018.

[93] J. Zhang, P. Cao, J. Gui et al., “Arctigenin ameliorates renal impairment and inhibits endoplasmic reticulum stress in diabetic db/db mice," Life Sciences, vol. 223, pp. 194-201, 2019.

[94] W. Wang, L. Qiu, A. Howard et al., "Protective effects of aliskiren and valsartan in mice with diabetic nephropathy," Journal of the Renin-Angiotensin-Aldosterone System, vol. 15, no. 4, pp. 384-395, 2014.

[95] J. C. Lim, S. K. Lim, M. J. Park, G. Y. Kim, H. J. Han, and S. H. Park, "Cannabinoid receptor 1 mediates high glucoseinduced apoptosis via endoplasmic reticulum stress in primary cultured rat mesangial cells," American Journal of Physiology. Renal Physiology, vol. 301, no. 1, pp. F179-F188, 2011.

[96] J. C. Lim, S. K. Lim, H. J. Han, and S. H. Park, “Cannabinoid receptor 1 mediates palmitic acid-induced apoptosis via endoplasmic reticulum stress in human renal proximal tubular cells," Journal of Cellular Physiology, vol. 225, no. 3, pp. 654-663, 2010.

[97] M. Kusaczuk, "Tauroursodeoxycholate-bile acid with chaperoning activity: molecular and cellular effects and therapeutic perspectives," Cell, vol. 8, no. 12, 2019.

[98] P. Zheng, Y. Lin, F. Wang et al., "4-PBA improves lithiuminduced nephrogenic diabetes insipidus by attenuating ER stress," American Journal of Physiology. Renal Physiology, vol. 311, no. 4, pp. F763-F776, 2016.

[99] Q. Guo, L. Xu, H. Li, H. Sun, S. Wu, and B. Zhou, "4-PBA reverses autophagic dysfunction and improves insulin sensitivity in adipose tissue of obese mice via Akt/mTOR signaling," Biochemical and Biophysical Research Communications, vol. 484, no. 3, pp. 529-535, 2017.

[100] X. Ji, L. Yao, M. Wang et al., "Cystatin C attenuates insulin signaling transduction by promoting endoplasmic reticulum stress in hepatocytes," FEBS letters, vol. 589, no. 24 Part B, pp. 3938-3944, 2015.

[101] A. L. Cao, L. Wang, X. Chen et al., "Ursodeoxycholic acid and 4-phenylbutyrate prevent endoplasmic reticulum stress- 
induced podocyte apoptosis in diabetic nephropathy," Laboratory Investigation, vol. 96, no. 6, pp. 610-622, 2016.

[102] L. Qin, Z. Wang, L. Tao, and Y. Wang, "ER stress negatively regulates AKT/TSC/mTOR pathway to enhance autophagy," Autophagy, vol. 6, no. 2, pp. 239-247, 2010.

[103] J. Y. Shih, Y. W. Lin, S. Fisch et al., "Dapagliflozin suppresses ER stress and improves subclinical myocardial function in diabetes: from bedside to bench," Diabetes, vol. 70, no. 1, pp. 262-267, 2021.

[104] E. Barreiro, A. Salazar-Degracia, A. Sancho-Muñoz, and J. Gea, "Endoplasmic reticulum stress and unfolded protein response profile in quadriceps of sarcopenic patients with respiratory diseases," Journal of Cellular Physiology, vol. 234, no. 7, pp. 11315-11329, 2019.

[105] K. R. Bohnert, J. D. McMillan, and A. Kumar, "Emerging roles of ER stress and unfolded protein response pathways in skeletal muscle health and disease," Journal of Cellular Physiology, vol. 233, no. 1, pp. 67-78, 2018.

[106] C. Y. Liu, C. C. Hsu, T. T. Huang et al., "ER stress-related ATF6 upregulates CIP2A and contributes to poor prognosis of colon cancer," Molecular Oncology, vol. 12, no. 10, pp. 1706-1717, 2018. 